\title{
Magnesium modulates the expression levels of calcification-associated factors to inhibit calcification in a time-dependent manner
}

\author{
JINSHENG XU, YALING BAI, JINGJING JIN, JUNXIA ZHANG, \\ SHENGLEI ZHANG, LIWEN CUI and HUIRAN ZHANG
}

Department of Nephrology, The Fourth Hospital of Hebei Medical University, Shijiazhuang, Hebei 050011, P.R. China

Received October 14 2014; Accepted December 15, 2014

DOI: $10.3892 /$ etm.2015.2215

\begin{abstract}
Vascular calcification, a common complication in patients with chronic kidney disease, involves a variety of mechanisms associated with the regulation of calcification-associated factors. Previous clinical studies have indicated that magnesium is involved in the reduction of vascular calcification; however, the mechanism underlying this process remains unknown. The aim of the present study was to investigate the effects of magnesium on $\beta$-glycerophosphate $(\beta-\mathrm{GP})$-induced calcification and the underlying mechanisms. Primary rat vascular smooth muscle cells (VSMCs) were exposed to $10 \mathrm{mM} \beta$-GP in medium with or without the addition of $3 \mathrm{mM}$ magnesium or 2-aminoethoxy-diphenylborate (2-APB; an inhibitor of magnesium transport), for a 14-day period. Calcium deposition and alkaline phosphatase (ALP) activity were measured by Alizarin red staining, quantification of calcium and enzyme-linked immunosorbent assay. The expression levels of core-binding factor $\alpha-1$ (Cbf $\alpha 1)$, matrix Gla protein (MGP) and osteopontin (OPN) were determined by reverse transcription-polymerase chain reaction or western blot analysis, following incubation for 0, 3, 6, 10 and 14 days with the different media. VSMC calcification and ALP activity was reduced significantly in the high-magnesium medium compared with the calcification medium, during the 14-day incubation. The magnesium-induced changes in the
\end{abstract}

Correspondence to: Dr Xu Jinsheng, Department of Nephrology, The Fourth Hospital of Hebei Medical University, 12 Jiankang Road, Shijiazhuang, Hebei 050011, P.R. China

E-mail: xjs5766@126.com

Abbreviations: 2-APB, 2-aminoethoxy-diphenylborate; ALP, alkaline phosphatase; $\beta$-GP, $\beta$-glycerophosphate; $\mathrm{Cbf} \alpha 1$, core binding factor $\alpha-1$; CKD, chronic kidney disease; MGP, matrix Gla protein; OPN, osteopontin; PBS, phosphate-buffered saline; TRPM7, transient receptor potential melastatin 7; VSMCs, vascular smooth muscle cells

Key words: vascular calcification, magnesium, core-binding factor $\alpha-1$, matrix Gla protein, osteopontin, time-dependent manner
VSMCs included a $\beta$-GP-induced downregulation of $\mathrm{Cbf} \alpha 1$ by day 3 of incubation, an effect that was gradually enhanced over the 14-day period. By contrast, magnesium produced notable increases in MGP and OPN expression levels, with an opposite pattern to that observed in the Cbf $\alpha 1$ expression levels. However, the addition of 2-APB appeared to inhibit the protective effect of magnesium on the VSMCs. Therefore, magnesium was able to effectively reduce $\beta$-GP-induced calcification in rat VSMCs by regulating the expression levels of calcification-associated factors in a time-dependent manner.

\section{Introduction}

Vascular calcification, a common phenomenon in patients with chronic kidney disease (CKD), is highly correlated with cardiovascular disease mortality (1-3). Prior studies have demonstrated that, in addition to traditional risk factors, nontraditional risk factors, including uremic toxin and a disturbed bone and mineral metabolism (in particular hyperphosphatemia) are considered to be associated with the high prevalence of vascular calcification in patients with CKD. Exposure of vascular smooth muscle cells (VSMCs) to high phosphate conditions results in notable mineralization with various putative processes, such as apoptosis and the osteogenic differentiation of VSMCs. Furthermore, high-phosphate conditions may lead to imbalance among the expression levels of calcification inducers, including core-binding factor $\alpha-1$ (Cbf $\alpha 1)$ and calcification inhibitors, such as matrix Gla protein (MGP) and osteopontin (OPN) (4-6). Although it has been studied extensively, there remains no effective treatment for vascular calcification.

Physiologically, magnesium is required for numerous fundamental functions in humans, including functioning as a 'natural competitor' of calcium and maintaining vascular tone and heart rhythm. Furthermore, a number of studies have demonstrated that low serum levels of magnesium may promote a number of metabolic syndromes, particularly type 2 diabetes mellitus, hypertension and atherosclerosis (7). Thus, magnesium may be clinically applicable due to its pleiotropic potential for interfering with vascular calcification. A study by Spiegel et al (8) indicated that magnesium carbonate inhibited the progression of coronary artery calcification for the 18-month duration of their pilot study. 
Subsequently, while reviewing the limited number of clinical studies on magnesium, Massy and Drüeke (9) identified a potential beneficial effect of magnesium in reducing vascular calcification and enhancing the survival rates of patients with CKD. In addition, it has been demonstrated at the cellular level that the addition of magnesium to a medium may reduce calcium deposition in cultured bovine VSMCs and in human aortic VSMCs (10). Furthermore, the preventative effect of magnesium in calcification is mitigated in the presence of 2-aminoethoxy-diphenylborate (2-APB), an inhibitor of transient receptor potential melastatin 7 (TRPM7), which is a transporter of $\mathrm{Mg}^{2+}(11)$. Magnesium has previously been indicated to modulate osteoblast differentiation of VSMCs in a dose-dependent manner (11). However, the mechanism underlying this magnesium-induced reduction in calcification remains unknown and requires further study.

The present study investigated the effects of magnesium on calcification and the expression levels of calcification-associated factors induced by $\beta$-glycerophosphate $(\beta$-GP) in rat VSMCs. The results suggested that magnesium inhibits $\beta$-GP-induced calcification in VSMCs by downregulating the expression of Cbfo1, while upregulating the expression of MGP and OPN in a time-dependent manner.

\section{Materials and methods}

Cell culture of VSMCs. Rat VSMCs were obtained from the tunica media of an adult male Sprague Dawley rat (Experimental Animal Center of Hebei Medical University, Shijiazhuang, China) thoracic aorta using the explant culture method as previously described (12) with a number of modifications as follows: Briefly, the rats were anesthetized with $400 \mathrm{mg} / \mathrm{kg}$ chloral hydrate (North China Pharmaceutical Limited by Share Ltd., Shijiazhuang, China) and the thoracic aorta was removed under aseptic conditions. The thoracic aorta was cut into $1-2-\mathrm{mm}^{2}$ pieces following the removal of any residual blood. The tissue pieces were cultured in dishes containing Dulbecco's modified Eagle's medium (DMEM; Gibco Life Technologies, Carlsbad, CA, USA) supplemented with $15 \%$ fetal bovine serum (FBS; Gibco Life Technologies), $4.5 \mathrm{~g}$ glucose, $100 \mathrm{U} / \mathrm{ml}$ penicillin and $100 \mu \mathrm{g} / \mathrm{ml}$ streptomycin (all from North China Pharmaceutical Limited by Share Ltd.) in a $5 \% \mathrm{CO}_{2}$ incubator at $37^{\circ} \mathrm{C}$. Cells that migrated from explants were collected when they reached $\sim 60-70 \%$ confluence. The cells were maintained in DMEM supplemented with $15 \%$ FBS, and the medium was replaced twice per week. VSMCs were identified by a positive staining of $\alpha$-smooth muscle actin (Sigma-Aldrich, St. Louis, MO, USA) and used for all the experiments between passages 3-4. The cells were analyzed following incubation for $0,3,6,10$ and 14 days. The current study was conducted in accordance with the Declaration of Helsinki (2013) and the Guide for Care and Use of Laboratory Animals as adopted and promulgated by the United National Institutes of Health (13). All experimental protocols were approved by the Review Committee for the Use of Animal Subjects of Hebei Medical University (Shijiazhuang, China).

Calcification assays. VSMC calcification was induced by incubation with a calcifying medium, which consisted of growth medium supplemented with $10 \mathrm{mM} \beta$-GP (Sigma-Aldrich). A high-magnesium medium was produced by adding $\mathrm{MgSO}_{4}$, with a final $\mathrm{Mg}^{2+}$ concentration of $3 \mathrm{mM}$. 2-APB (Sigma-Aldrich), the TRPM7 inhibitor, was added to reach a final concentration of $10^{-4} \mathrm{M}$. Following incubation for 14 days, cells were washed twice with phosphate-buffered saline (PBS; Beijing Solarbio Science \& Technology Company Co., Ltd., Beijing, China) and fixed with $95 \%$ ethanol. The cells were exposed to $0.2 \%$ Alizarin red $(\mathrm{pH} 8.3$; Beijing Solarbio Science \& Technology Company Co., Ltd.). Subsequent to washing with PBS, the cells were visualized and images were captured to record the incidence of induced calcification by an inverted phase contrast microscope (type LH50A; Olympus Corporation, Tokyo, Japan). The software used to capture images was NIS-Element F3.0 (Olympus Corporation). Subsequently, calcium deposited in the extracellular matrix was extracted with $0.6 \mathrm{M} \mathrm{HCl}$ for $24 \mathrm{~h}$ at $37^{\circ} \mathrm{C}$. The calcium content in the supernatant was measured with the o-cresolphthalein complexone method using a Calcium Assay kit according to the manufacturer's instructions (BioSino Biotechnology \& Science, Inc., Beijing, China) and normalized relative to the protein concentration of the same culture.

Alkaline phosphatase (ALP) activity. The cells were cultured for 14 days following treatment. ALP activity was measured using an Alkaline Phosphatase Activity Detection kit (Nanjing Jiancheng Bioengineering Institute, Nanjing, China) in accordance with the manufacturer's instructions. Cell protein content was measured with a bicinchoninic acid (BCA) protein assay kit (Beijing Solarbio Science \& Technology Company Co., Ltd.) and ALP activity was normalized against the total protein.

Reverse transcription-quantitative polymerase chain reaction (RT-qPCR) to assess levels of Cbfal, MGP and OPN expression. The target genes Cbf $\alpha 1$, MGP and OPN were determined by RT-qPCR, performed using a PCR Master Mix kit (Promega Corporation, Madison, WI, USA) following incubation for $0,3,6,10$ and 14 days. The GAPDH gene was used as an endogenous control. The primer sequences used for PCR amplification were as follows: Rat Cbf $\alpha 1$, F 5'-CCG CAC GAC AAC CGC ACC AT-3' and R 5'-CGC TCC GGC CCA CAA ATC TC-3' (generating an amplified fragment of $289 \mathrm{bp}$ ); rat MGP, F 5'-AAA GCC CAG GAA AGA GTC CG-3' and R 5'-TCT TAT TTG GCT CCT CGG CG-3' (generating an amplified fragment of $158 \mathrm{bp}$ ); rat OPN, F 5'-ATG CTA TCG ACA GTC AGG CG-3' and R 5'-GCT CAG GGC CCA AAA CAC TA-3' (generating an amplified fragment of $317 \mathrm{bp}$ ); rat GAPDH, F 5'-CCC ACT AAA GGG CAT CCT GG-3' and R 5'-GGC CCC TCC TGT TGT TAT GG-3' (generating an amplified fragment of 352 bp). The PCR products $(5 \mu \mathrm{l})$ were subjected to electrophoresis (MultiSUB Midi-96; Beijing Thmorgan Biotechnology Co., Ltd., Beijing, China) using a $1.5 \%$ agarose gel and visualized with an ethidium bromide stain (all from Invitrogen Life Technologies, Carlsbad, CA, USA). The band optical density was measured using a Gel Documentation System (CST Biological Reagents Company Limited, Shanghai, China) and the final data are expressed as the mRNA level relative to that of GAPDH. 


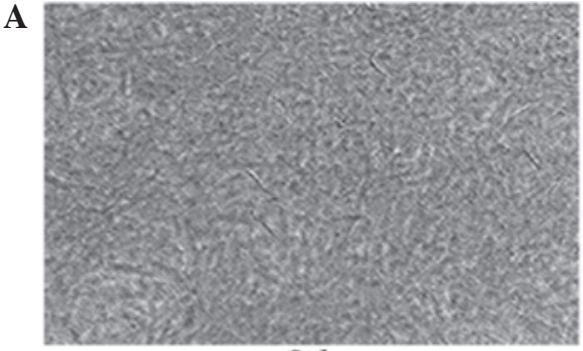

$\mathrm{Ct} 1$

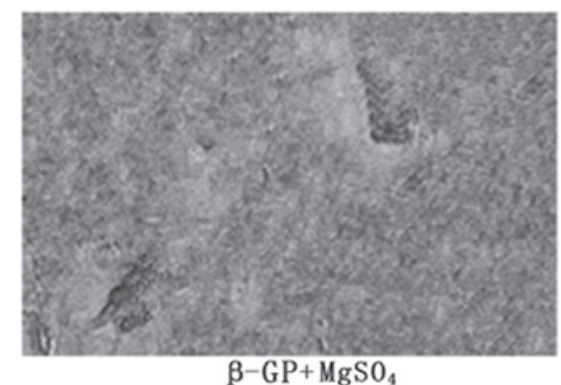

B

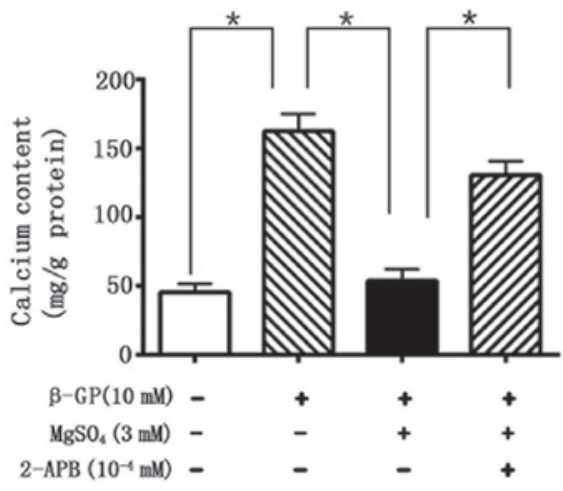

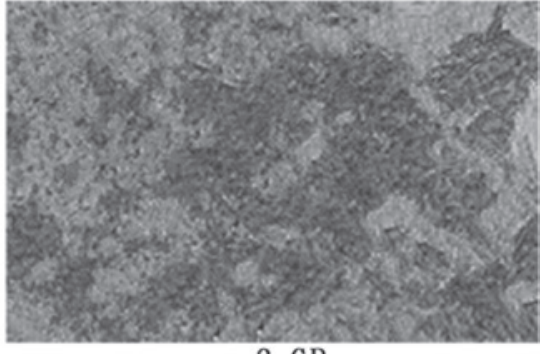

B-GP

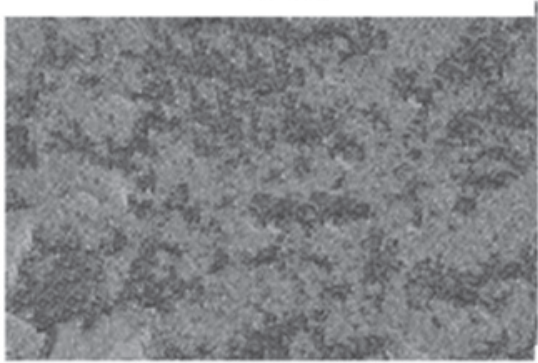

$\beta-\mathrm{GP}+\mathrm{MgSO}_{4}+2-\mathrm{APB}$

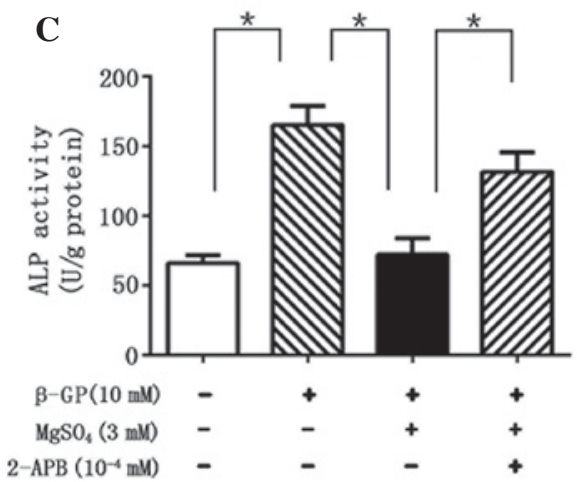

Figure 1. (A) Alizarin red staining was performed on cells incubated under different conditions for 14 days. (B) Calcium content and (C) ALP activity are expressed as the mean \pm standard deviation of at least three simultaneous replicates. Ctl, control; $\beta$-GP, $\beta$-glycerophosphate; 2 -APB, 2 -aminoethoxy-diphenylborate; ALP, alkaline phosphatase.

Western blot analysis of Cbfal protein expression. Total protein was extracted from the VSMCs, and the concentrations were measured with the BCA protein assay kit following incubation for $0,3,6,10$ and 14 days. The protein samples were resolved on a $10 \%$ Tris/glycine SDS-polyacrylamide gel (Invitrogen Life Technologies) in running buffer containing $25 \mathrm{mmol} / \mathrm{l}$ Tris, $192 \mathrm{mmol} / \mathrm{l}$ glycine and $0.1 \%$ SDS. The proteins were then transferred to a nitrocellulose membrane for $3 \mathrm{~h}$ at $4^{\circ} \mathrm{C}$ at $300 \mathrm{~mA}$ in a transfer buffer containing $20 \mathrm{mmol} / \mathrm{l}$ Tris- $\mathrm{HCl}$ ( $\mathrm{pH}$ 8.0; Invitrogen Life Technologies), $150 \mathrm{mmol} / \mathrm{l}$ glycine and $20 \%$ methanol. Non-specific protein binding was blocked by incubating the membrane with 5\% non-fat dry milk in TBS-T [20 mmol/1 Tris- $\mathrm{HCl}(\mathrm{pH} 7.6$ ), $150 \mathrm{mmol} / 1 \mathrm{NaCl}$ and $0.02 \%$ Tween 20; Invitrogen Life Technologies] for $1 \mathrm{~h}$ at room temperature with agitation. A monoclonal mouse anti-mouse $\mathrm{Cbf} \alpha 1$ primary antibody (Sigma-Aldrich, St. Louis, MO, USA) was added to the membrane at a 1:500 dilution in TBS-T and incubated at $4^{\circ} \mathrm{C}$ overnight with agitation. The monoclonal rabbit antimouse secondary antibody (Sigma-Aldrich) was diluted in TBS-T (1:2,000 dilution) and applied to the membrane, and the reaction was incubated at room temperature for $1 \mathrm{~h}$ with agitation. Between each of the three proceeding steps (primary antibody, secondary antibody and visualization) the membrane was washed 3 times for 10 min each time with TBS-T at room temperature. The membrane was visualized and analyzed using a Chemiluminescence Imaging system (CST Biological Reagents Company Limited). The $\beta$-actin (42 kDa; Sigma-Aldrich) protein was used as an endogenous control.

Statistical analysis. Data analyses were conducted using SPSS 19.0 software (SPSS, Inc., Chicago, IL, USA). All results were expressed as mean \pm standard deviation. Differences among groups were determined by analysis of variance (ANOVA), and the Student-Newman-Keuls method was used for post-hoc testing. $\mathrm{P}<0.05$ denoted a statistically significant difference.

\section{Results}

Magnesium attenuates $\beta$-GP-induced calcification in VSMCs. The calcification of VSMCs was assessed under different conditions in order to evaluate the effects of magnesium on the process. VSMCs incubated with calcifying medium ( $\beta$-GP) for 14 days exhibited evident calcification, observed by Alizarin red staining, compared with the control cells (Fig. 1A). However, these changes were clearly reversed in the cells maintained in the high-magnesium medium. 
A

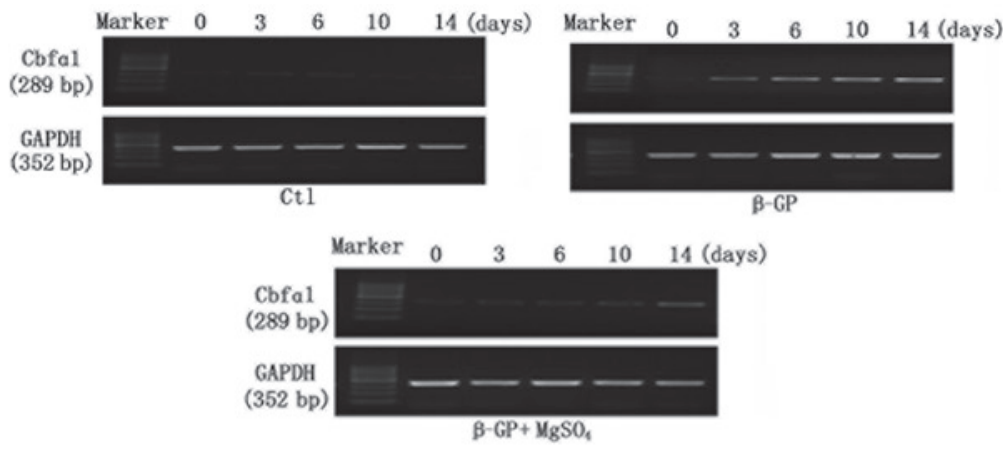

C
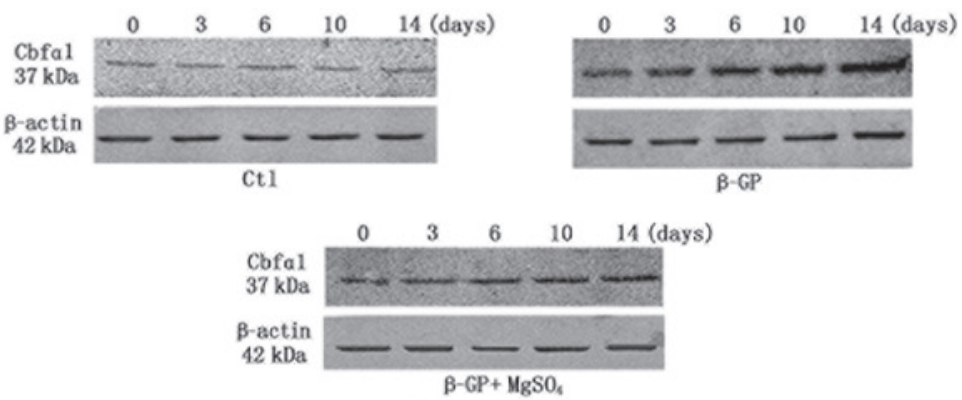

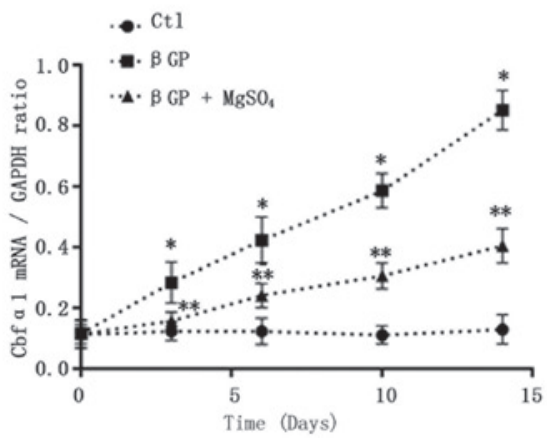

D

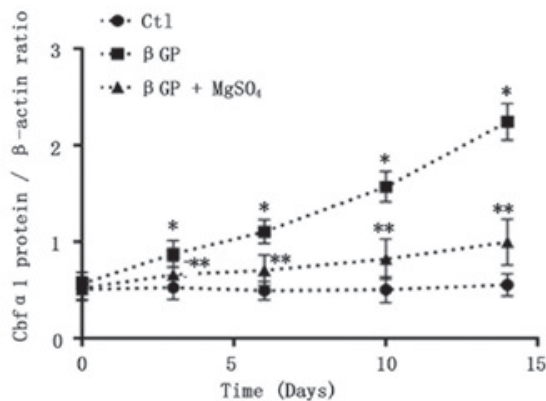

Figure 2. Relative expression levels of Cbf $1 \mathrm{mRNA}$ and protein. (A) Representative Cbf $\alpha 1 \mathrm{mRNA}$ expression levels from cells following treatment with control medium, $\beta$-GP or $\beta$-GP $+\mathrm{MgSO}_{4}$ for 0-14 days. (B) Quantification of relative expression levels of Cbfa1. (C) Representative western blots of Cbf $\alpha 1$ protein in the cells. (D) Quantification of relative protein levels of Cbf $\alpha 1$. Cbf $\alpha 1$, core-binding factor $\alpha-1$; Ct1, control; $\beta$-GP, $\beta$-glycerophosphate.

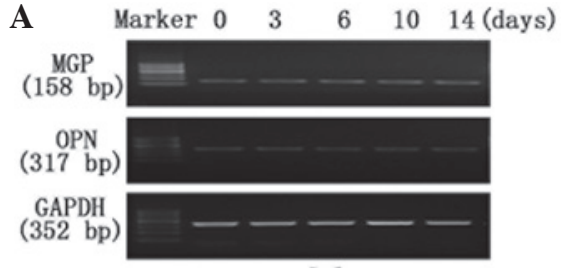

Ct1

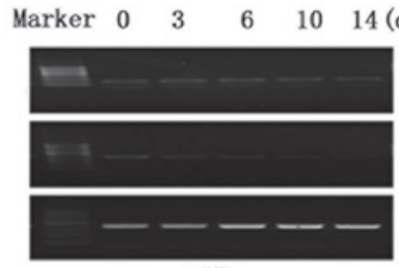

$\beta-$ GP

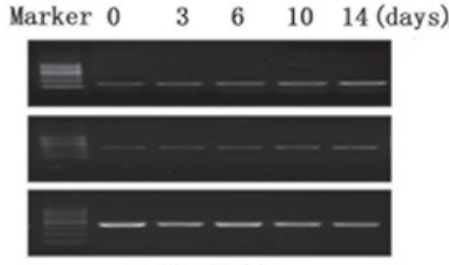

$\mathrm{B}-\mathrm{GP}+\mathrm{MgSO}_{4}$
B

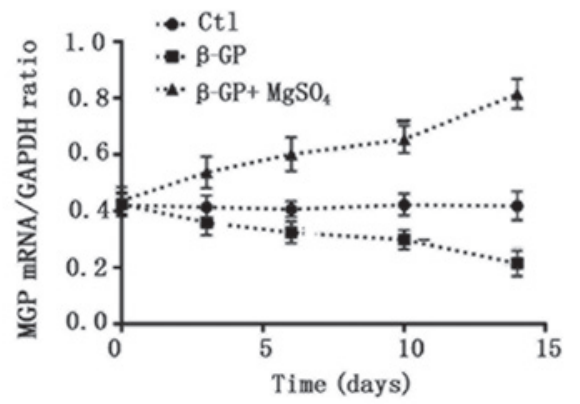

C

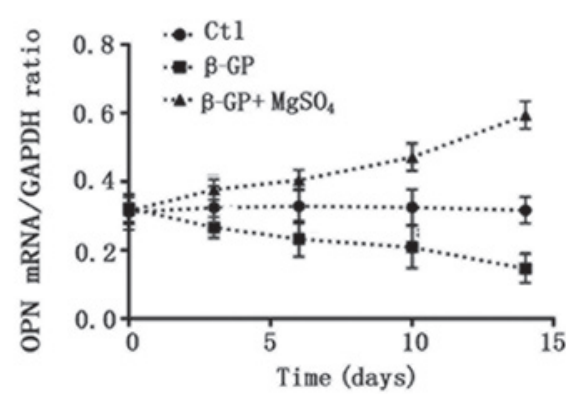

Figure 3. Relative expression levels of MGP and OPN mRNA. (A) Representative mRNA expression following treatment with control medium, $\beta$-GP or $\beta-\mathrm{GP}+\mathrm{MgSO}_{4}$ for 0-14 days. Quantification of the relative mRNA expression levels of (B) MGP and (C) OPN. MGP, matrix Gla protein; OPN, osteopontin; Cbf $\alpha 1$; Ctl, control; $\beta$-GP, $\beta$-glycerophosphate.

In agreement, quantitative analysis indicated that calcium content in the VSMCs was significantly reduced under high-magnesium conditions compared with calcifying conditions (Fig. 1B). Furthermore, ALP activity, a vital marker of calcification, was markedly enhanced following $\beta$-GP treatment, while inhibited in the presence of magnesium (Fig. 1C). The reduction in calcium content was significantly counteracted by the addition of 2-APB, indicating that the anti-calcification effect was mediated by the magnesium.
Magnesium inhibits $\beta$-GP-induced osteoblastic factor expression of VSMCs in a time-dependent manner. It is established that under calcifying conditions, VSMCs enhance mineralization through the passive deposition of calcium-phosphate, and also by active transformation into osteoblast-like cells, which is a strictly regulated cellular process that is similar to bone formation. Thus, the effects of long-term (14-day) calcification culture on $\mathrm{Cbf} \alpha 1$ with or without $3 \mathrm{mM}$ magnesium were assessed. As expected, on the third day the levels of Cbf $\alpha 1$ 
A

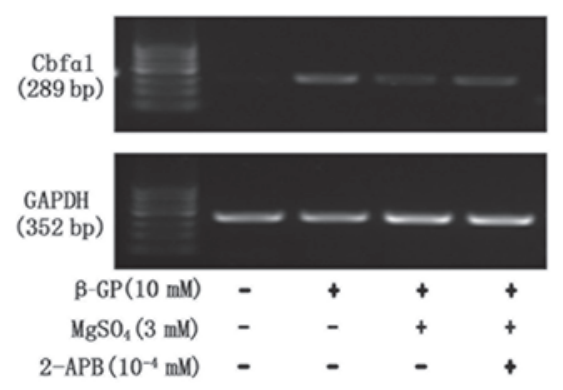

C

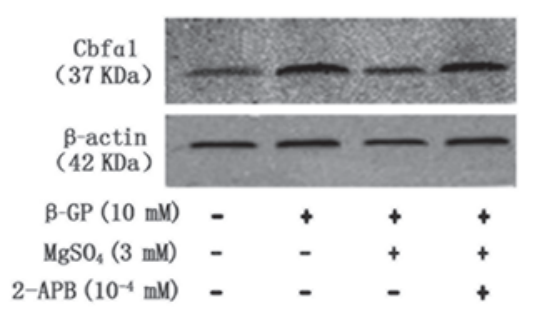

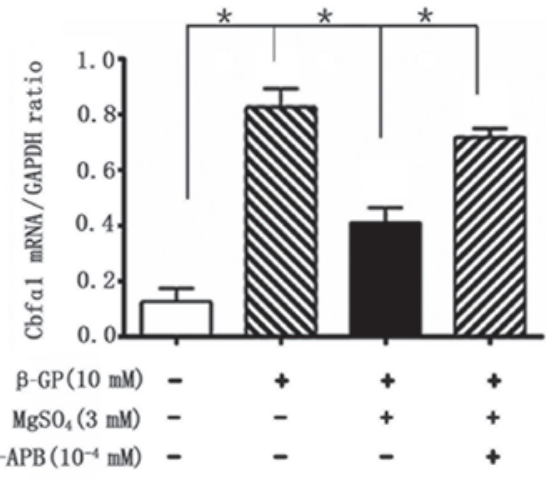

D

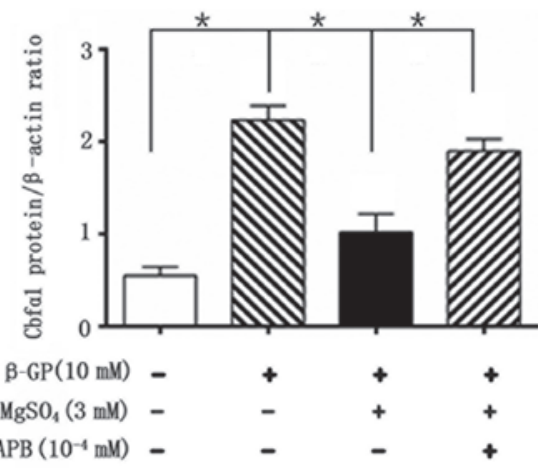

Figure 4. 2-APB inhibits the effect of magnesium on Cbf $\alpha 1$ expression levels. (A) Representative mRNA levels of Cbf $\alpha 1$ in cells following treatment with $\beta$-GP, $\mathrm{MgSO}_{4}$ and 2-APB for 14 days. (B) Quantification of the relative mRNA expression levels of Cbf $\alpha 1$. (C) Representative western blot analysis of Cbfal in cells following treatment with $\beta$-GP, $\mathrm{MgSO}_{4}$ and 2-APB for 14 days. (D) Quantification of the relative protein levels of Cbf $\alpha 1$. Cbf $\alpha 1$, core-binding factor $\alpha-1 ; \beta$-GP, $\beta$-glycerophosphate; 2-APB, 2-aminoethoxy-diphenylborate.

A

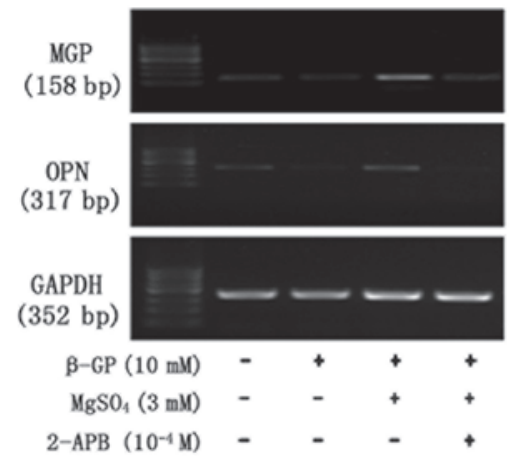

B

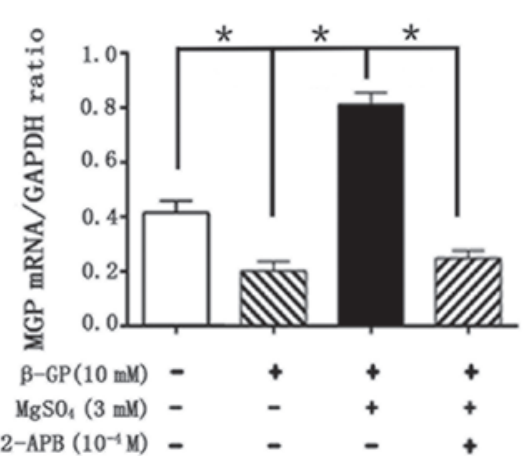

C

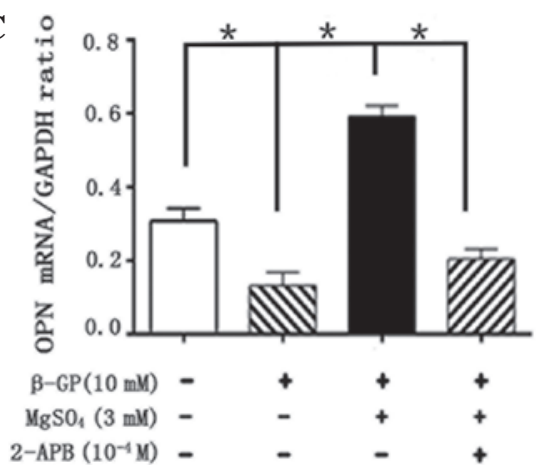

Figure 5. 2-APB inhibits the effect of magnesium on the expression of MGP and OPN. (A) Representative mRNA levels of MGP and OPN in cells following treatment with $\beta$-GP, $\mathrm{MgSO}_{4}$ and 2-APB for 14 days. Quantification of the relative mRNA expression levels of (B) MGP and (C) OPN. MGP, matrix Gla protein; OPN, osteopontin; $\beta$-GP, $\beta$-glycerophosphate; 2-APB, 2-aminoethoxy-diphenylborate.

mRNA (Figs. 2A and B) and protein (Fig. 2C and D) began to increase significantly and a notable time-dependent increase in the Cbf $\alpha 1$ expression level was observed in the VSMCs incubated with calcifying medium ( $\beta$-GP). Short-term (3-day) incubation in the high-concentration magnesium medium markedly reduced $\beta$-GP-induced Cbf $\alpha 1$ expression, with the effect remaining enhanced throughout the culture period (Fig. 2).

Magnesium regulates the secretion of calcification inhibitors in a time-dependent manner. Under physiological conditions, VSMCs may mitigate calcification through a variety of processes. To date, studies have demonstrated that the vascular calcification process involves an imbalance of inducers and inhibitors (14). In the present study, it was established whether the magnesium modulation of $\mathrm{Cbf} \alpha 1$ levels resulted in alterations in the expression levels of MGP and OPN (inhibitors of calcification) in a 14-day period. RT-qPCR indicated that $\beta$-GP treatment led to a significant reduction in the levels of MGP and OPN by day 3 of incubation, and that this reduction was time-dependent. Incubation for 3 days in $\beta$-GP with magnesium inhibited the $\beta$-GP-induced reduction in the levels of MGP and OPN. Furthermore, long-term (14-day) exposure to the high-magnesium environment restored, and also gradually upregulated, the expression levels of MGP and OPN (Fig. 3).

2-APB inhibits the effects of magnesium on VSMC calcification and associated gene expression levels. To clarify the 
protective molecular mechanisms of magnesium on VSMC calcification, the role of TRPM7 in the magnesium-induced regulation of calcification was investigated. VSMCs treated with 2-APB, a specific inhibitor of TRPM7, exhibited no magnesium-induced reduction in calcification, and recovered the magnesium-inhibited decrease of ALP activity by day 14 (Fig. 1). Furthermore, 2-APB reversed the magnesium-induced reduction in Cbfol mRNA (Fig.4A and $\mathrm{B}$ ) and protein (Fig. 4C and D) expression levels and the magnesium-induced increase in the mRNA expression levels of MGP and OPN, following 14 days of incubation (Fig. 5).

\section{Discussion}

Vascular calcification is a notable risk factor for mortality in cardiovascular diseases, particularly in patients with CKD (15). Although previous studies have demonstrated that numerous factors are involved in the regulation of vascular calcification, an effective therapy has yet to be developed (14). A number of studies have indicated that magnesium has the potential to prevent vascular calcification $(7,8)$. Clinical studies have suggested that calcification and carotid intima media thickness increase are strongly associated with low magnesium levels $(16,17)$, whereas supplementation of magnesium may reduce carotid intima media thickness and retard arterial calcification in patients with CKD (18). However, there are limited studies investigating the effect of magnesium on vascular calcification in vitro. The present study investigated the action of magnesium on $\beta$-GP-induced calcification processes in rat VSMCs. The magnesium concentration used in the current study was based on the high-magnesium plasma levels observed in patients with CKD, which range between 2.5 and $3 \mathrm{mmol} / \mathrm{l}$ (19). As predicted, it was identified that the addition of magnesium to the medium markedly inhibited $\beta$-GP-induced calcification and ALP activity by day 14 . Therefore, the results further confirmed that magnesium serves an important function in the prevention of the $\beta$-GP-induced calcification of VSMCs, and may be useful in the treatment of vascular calcification.

However, the mechanisms of the magnesium-induced reduction of calcification remain unclear. Prior studies suggest that vascular calcification is an active, cell-mediated process, which may lead to the osteogenic differentiation of VSMCs and subsequent extracellular matrix mineralization (20). A number of studies have demonstrated that magnesium is able to interfere with VSMC differentiation, possibly in a dose-dependent manner $(21,22)$. In support of these findings, the present study assessed the expression of $\mathrm{Cbf} \alpha 1$, a central transcription factor for bone formation, osteogenic matrix gene expression, VSMC differentiation and calcification. The increase in phosphate concentration in vitro results in the downregulation of smooth muscle-specific gene expression and the upregulation of $\mathrm{Cbf} \alpha 1$ expression (23). Furthermore, $\mathrm{Cbf} \alpha 1$ has been demonstrated to be expressed in the calcified vascular lesions of patients with CKD (24). Thus, Cbf $\alpha 1$ has been considered as the earliest and most specific osteogenic differentiation marker for the promotion of calcification. In the present study, the expression of Cbf $\alpha 1$ was markedly upregulated by treatment with $\beta$-GP for 14 days. This phenomenon was inhibited in cells that were co-cultured in a medium with a high magnesium concentration. Furthermore, it was demonstrated for the first time that magnesium downregulates the $\beta$-GP-induced expression of Cbf $\alpha 1$. This effect became increasingly evident over the duration of the incubation, suggesting that magnesium reduced vascular calcification partly by reversing the transdifferentiation of VSMCs under calcifying conditions in a time-dependent manner.

MGP, an established inhibitor of vascular calcification, is expressed in normal cartilage and cardiovascular tissues, but is downregulated in atherosclerotic tissue (22). The knockout of MGP has been associated with extensive, spontaneous calcification and premature mortality in mice (25). The protective effects of MGP have been hypothesized to be partly associated with the inhibition of bone morphogenetic protein-2-induced cell differentiation (26), which is pivotal in Cbfol expression and bone formation. Similarly to MGP, OPN is an inhibitor of calcification, and is directly associated with bone resorption, which is activated by the parathyroid hormone-receptor activator of nuclear factor- $\kappa \mathrm{B}$ ligand axis in the bone (27). The double-knockout of MGP and OPN in mice has been demonstrated to result in more severe vascular calcification compared with MGP-only knockout mice. The inhibitory effect of OPN has been partly attributed to it binding tightly to hydroxyapatite (27) and actively inducing decalcification (28). In the present study, MGP and OPN levels were restored and also notably upregulated in VSMCs incubated in a high-magnesium medium for 14 days. Furthermore, it was demonstrated for the first time that the magnesium-induced increase in the expression levels of MGP and OPN occurred in a time-dependent manner. As indicated by the alteration in Cbf $\alpha 1$, magnesium inhibited calcification partly by gradually restoring the balance between inducer and inhibitor regulatory proteins.

In addition to this finding, high concentration magnesium has been indicated to prevent $\beta$-GP-induced cell apoptosis (11). Magnesium has also been demonstrated to reduce vascular calcification in live VSMCs, but not in fixed cells (10). These findings, together with the results of the current study, suggest that magnesium serves an active role in vascular calcification. VSMCs were exposed to 2-APB, an inhibitor of the magnesium transporter TRPM7, in order to test more detailed molecular mechanisms underlying the active effects of magnesium on calcification $(29,30)$. The beneficial effects of magnesium on calcification were inhibited by the addition of $2-\mathrm{APB}$ at $10^{-4} \mathrm{M}$. At this concentration, 2-APB efficiently inhibited the effect of magnesium on $\beta$-GP-induced overexpression of osteogenic transcription factor $\mathrm{Cbf} \alpha 1$. In accordance with previous studies, the inhibition of TRPM7 by 2-APB prevents the transport of magnesium, and results in the loss of the protective effect of magnesium on calcification inhibitors. These findings suggested that the inhibitory effect of magnesium on calcification is an active, intracellular process. However, the effect of 2-APB on TRPM7 was not time-dependent. Thus, further investigations are required to clarify the role of TRPM7 in the regulation of magnesium homeostasis.

In conclusion, magnesium was able to efficiently reduce $\beta$-GP-induced calcification in rat VSMCs. Furthermore, magnesium inhibited the transdifferentiation of VSMCs into osteoblast-like cells by reducing the expression of $\mathrm{Cbf} \alpha 1$ in a time-dependent manner. In addition, magnesium was 
observed to upregulate the expression levels of the inhibitors MGP and OPN in a time-dependent manner. These findings suggest that magnesium reduces mineralization by regulating the expression levels of calcification-associated factors in a time-dependent manner. However, further studies are required to clarify the mechanism underlying the magnesium-induced inhibition of vascular calcification.

\section{Acknowledgements}

The present study was supported by the project of the Hebei Natural Science Fund (no. H2012206157) and the project of the Hebei Major Medical Science (no. GL2011-51).

\section{References}

1. Foley RN and Parfrey PS: Cardiovascular disease and mortality in ESRD. J Nephrol 11: 239-245, 1998.

2. Goodman WG, Goldin J, Kuizon BD, et al: Coronary-artery calcification in young adults with end-stage renal disease who are undergoing dialysis. N Engl J Med 342: 1478-1483, 2000

3. Blacher J, Guerin AP, Pannier B, Marchais SJ and London GM: Arterial calcifications, arterial stiffness, and cardiovascular risk in end-stage renal disease. Hypertension 38: 938-942, 2001.

4. Scatena M, Liaw L and Giachelli CM: Osteopontin: a multifunctional molecule regulating chronic inflammation and vascular disease. Arterioscler Thromb Vasc Biol 27: 2302-2309, 2007.

5. O'Neill WC: Mineral complexes and vascular calcification. Kidney Int 76: 915-916, 2009.

6. Zarjou A, Jeney V, Arosio P, et al: Ferritin prevents calcification and osteoblastic differentiation of vascular smooth muscle cells. J Am Soc Nephrol 20: 1254-1263, 2009.

7. Geiger $\mathrm{H}$ and Wanner C: Magnesium in disease. Clin Kidney J 5 (Suppl 1): i25-i38, 2012.

8. Spiegel DM and Farmer B: Long-term effects of magnesium carbonate on coronary artery calcification and bone mineral density in hemodialysis patients: a pilot study. Hemodial Int 13 453-459, 2009

9. Massy ZA and Drüeke TB: Magnesium and outcomes in patients with chronic kidney disease: focus on vascular calcification, atherosclerosis and survival. Clin Kidney J 5 (Suppl 1): i52-i61, 2012.

10. Louvet L, Büchel J, et al: Magnesium prevents phosphate-induced calcification in human aortic vascular smooth muscle cells. Nephrol Dial Transplant 28: 869-878, 2013.

11. Kircelli F, Peter ME, Sevinc Ok E, et al: Magnesium reduces calcification in bovine vascular smooth muscle cells in a dose-dependent manner. Nephrol Dial Transplant 27: 514-521, 2012.

12. Cai Y, Teng X, Pan CS, Duan XH, Tang CS and Qi YF: Adrenomedullin up-regulates osteopontin and attenuates vascular calcification via the cAMP/PKA signaling pathway. Acta Pharmacol Sin 31: 1359-1366, 2010.

13. Carbone L: Pain management standards in the eighth edition of the Guide for the Care and Use of Laboratory Animals. J Am Assoc Lab Anim Sci 51: 322-328, 2012.
14. Mizobuchi M, Towler D and Slatopolsky E: Vascular calcification: the killer of patients with chronic kidney disease. J Am Soc Nephrol 20: 1453-1464, 2009.

15. Pun PH, Smarz TR, Honeycutt EF, Shaw LK, Al-Khatib SM and Middleton JP: Chronic kidney disease is associated with increased risk of sudden cardiac death among patients with coronary artery disease. Kidney Int 76: 652-658, 2009.

16. Tzanakis I, Pras A, Kounali D, et al: Mitral annular calcifications in haemodialysis patients: a possible protective role of magnesium. Nephrol Dial Transplant 12: 2036-2037, 1997.

17. Tzanakis I, Virvidakis K, Tsomi A, et al: Intra- and extracellular magnesium levels and atheromatosis in haemodialysis patients. Magnes Res 17: 102-108, 2004.

18. Turgut F, Kanbay M, Metin MR, Uz E, Akcay A and Covic A: Magnesium supplementation helps to improve carotid intima media thickness in patients on hemodialysis. Int Urol Nephrol 40: 1075-1082, 2008

19. Montezano AC, Zimmerman D, Yusuf H, et al: Vascular smooth muscle cell differentiation to an osteogenic phenotype involves TRPM7 modulation by magnesium. Hypertension 56: 453-462, 2010.

20. Jono S, McKee MD, Murry CE, et al: Phosphate regulation of vascular smooth muscle cell calcification. Circ Res 87: E10-E17, 2000.

21. Sun Y, Byon CH, Yuan K, et al: Smooth muscle cell-specific runx2 deficiency inhibits vascular calcification. Circ Res 111: 543-552, 2012.

22. Zhong H, Liu F, Dai X, Zhou L and Fu P: Sodium thiosulfate protects human aortic smooth muscle cells from osteoblastic transdifferentiation via high-level phosphate. Kaohsiung J Med Sci 29: 587-593, 2013.

23. Steitz SA, Speer MY, Curinga G, et al: Smooth muscle cell phenotypic transition associated with calcification: upregulation of Cbfal and downregulation of smooth muscle lineage markers. Circ Res 89: 1147-1154, 2001

24. Moe SM, Duan D, Doehle BP, O'Neill KD and Chen NX: Uremia induces the osteoblast differentiation factor Cbfa1 in human blood vessels. Kidney Int 63: 1003-1011, 2003.

25. Luo G, Ducy P, McKee MD, et al: Spontaneous calcification of arteries and cartilage in mice lacking matrix GLA protein. Nature 386: 78-81, 1997.

26. Boström K, Tsao D, Shen S, Wang Y and Demer LL: Matrix GLA protein modulates differentiation induced by bone morphogenetic protein-2 in C3H10T1/2 cells. J Biol Chem 276: 14044-14052, 2001.

27. Wada T, McKee MD, Steitz S and Giachelli CM: Calcification of vascular smooth muscle cell cultures: inhibition by osteopontin. Circ Res 84: 166-178, 1999.

28. Speer MY, McKee MD, Guldberg RE, et al: Inactivation of the osteopontin gene enhances vascular calcification of matrix Gla protein-deficient mice: evidence for osteopontin as an inducible inhibitor of vascular calcification in vivo. J Exp Med 196: 1047-1055, 2002.

29. He Y, Yao G, Savoia C and Touyz RM: Transient receptor potential melastatin 7 ion channels regulate magnesium homeostasis in vascular smooth muscle cells: role of angiotensin II. Circ Res 96: 207-215, 2005.

30. Paravicini TM, Chubanov V and Gudermann T: TRPM7: a unique channel involved in magnesium homeostasis. Int J Biochem Cell Biol 44: 1381-1384, 2012. 